\title{
Várices esofágicas superiores (Downhill varices). Presentación de casos y revisión de la literatura
}

\section{Upper esophageal varices (Downhill varices): Case presentation and literature review}

Luis Alberto Ángel Arango, MD, ${ }^{1}$ Andrés Felipe Donado Moré, MD. ${ }^{2 *}$

\footnotetext{
1 Unidad de Gastroenterología. Hospital Universitario Nacional. Facultad de Medicina. Departamento de Medicina Interna. Universidad Nacional de Colombia - Gastroinvest S.A.S. IDIME. Bogotá D.C., Colombia. 2 Departamento de Radiología e Imágenes Diagnósticas. Hospital Universitario Nacional. Departamento de Radiología e Imágenes Diagnósticas. Facultad de Medicina. Universidad Nacional de Colombia. Bogotá, Colombia.

*Correspondencia: Andrés Felipe Donado Moré, andresdonado@live.com

Fecha recibido: $11 / 03 / 19$ Fecha aceptado: 29/07/19
}

\begin{abstract}
Resumen
Se describen 21 casos con hallazgo incidental de várices esofágicas superiores (Downhill varices) en endoscopia de las vías digestivas altas. Además, se detallan algunos datos relevantes de la historia clínica y de los hallazgos endoscópicos. La relación hombres/mujeres fue 1:1 y la edad promedio fue de 67,9 años. La comorbilidad más frecuente fue la enfermedad pulmonar obstructiva crónica (EPOC), seguida de las neumoconiosis, la obesidad y el síndrome de apnea-hipopnea, todas con hipertensión pulmonar (HTP) asociada. Las tres últimas causas no han sido descritas previamente en la literatura sobre várices esofágicas superiores. Ningún paciente tenía antecedente de hemorragia variceal.
\end{abstract}

\section{Palabras clave}

Várices esofágicas superiores, Downhill varices, descripción de casos, revisión, hipertensión pulmonar (HTP).

\section{Abstract}

We describe 21 cases with incidental endoscopic findings of upper esophageal varices (downhill varices) and relevant data from patients' clinical histories and endoscopic findings. The male/female ratio was 1:1 and patients' average age was 67.9 years. The most frequent comorbidity was chronic obstructive pulmonary disease, followed by pneumoconiosis, obesity and apnea-hypopnea syndrome. All comorbidities had associated pulmonary hypertension. The last three causes have not been previously described in the literature on upper esophageal varices. None of the patients had histories of variceal hemorrhaging.

\section{Keywords}

Upper esophageal varices, downhill varices, case description, review, pulmonary hypertension.

\section{INTRODUCCIÓN}

Las várices esofágicas superiores son un hallazgo endoscópico infrecuente que se puede encontrar de forma incidental o como parte del estudio de una hemorragia de las vías digestivas altas. A diferencia de las várices esofágicas inferiores, estas son secundarias a trastornos en el retorno venoso desde los tercios superior y medio del esófago hacia la vena cava superior (VCS), lo que determina su nombre de Downhill en la literatura en inglés.
La causa más frecuente en las descripciones iniciales fueron las obstrucciones por tumores mediastinales o la fibrosis del mediastino (1), que luego fueron superadas por los fenómenos trombóticos de la VCS y sus afluentes, dado el creciente uso de dispositivos intravasculares (2).

Este estudio corresponde a una serie de casos en los que se detectaron várices esofágicas superiores, a través de una endoscopia de las vías digestivas altas, de variadas etiologías y diferentes a las clásicas antes mencionadas. Se describen sus características clínicas y la morbilidad asociada, 
así como también se realiza una revisión bibliográfica y se comparan los casos con los descritos en la literatura.

\section{SUJETOS Y MÉTODOS}

Se describe una serie de casos de 21 pacientes que acudieron a unidades de endoscopia de vías digestivas superiores, ubicadas en Bogotá (Colombia). Como método de diagnóstico se empleó la videoendoscopia de vías digestivas superiores. En este trabajo, se detallan las características demográficas, el diagnóstico y las características clínicas -cuando estas estuvieron disponibles- y los hallazgos endoscópicos documentados con videos y fotografías.

Los resultados fueron tabulados a partir de medidas paramétricas y no paramétricas. Además, se realizó una revisión bibliográfica, a través de una búsqueda efectuada en PubMed, para lo cual se usó el término Downhill varices (detalles de la estrategia: Downhill [All Fields] AND "varicose veins" [MeSH Terms] OR "varicose" [All Fields] AND "veins" [All Fields]) OR "varicose veins" [All Fields] OR "varices" [All Fields]) y a partir de la bibliografía de los artículos originales sobre el tema.

\section{DESCRIPCIÓN DE LOS CASOS}

En primer lugar, se describirán los casos relevantes, con datos clínicos y documentos fotográficos. Luego se presentarán los hallazgos generales de todos los casos en su orden de descripción cronológica (Tablas 1 y 2).

Tabla 1. Descripción de casos de pacientes con várices esofágicas proximales

\begin{tabular}{|c|c|c|c|c|c|c|c|c|}
\hline \multirow[t]{2}{*}{ Caso } & \multirow[t]{2}{*}{ Paciente $^{*}$} & \multirow[t]{2}{*}{ Edad } & \multirow[t]{2}{*}{ Sexo** } & \multirow{2}{*}{$\begin{array}{l}\text { Diagnóstico } \\
\text { clínico }^{+}\end{array}$} & \multicolumn{3}{|c|}{ Várices } & \multirow[t]{2}{*}{ Enfermedades de base ${ }^{++}$} \\
\hline & & & & & Proximales & Distales & Fúndicas & \\
\hline 1 & AS & 57 & $\mathrm{~F}$ & & Sí & No & No & \\
\hline 2 & AJOC & 48 & $\mathrm{~F}$ & $\begin{array}{l}\text { RGE - Masa } \\
\text { epigástrica }\end{array}$ & Sí & No & No & $\begin{array}{l}\text { Cirugía bariátrica, suspensión CPAP, hidronefrosis por } \\
\text { litiasis derecha, RGE por gammagrafía. }\end{array}$ \\
\hline 2 & AJOC & 42 & $\mathrm{~F}$ & RGE - HH & Sí & No & No & $\begin{array}{l}\text { Masa en epigastrio, obesidad mórbida, apnea del sueño, } \\
\text { CPAP, SAOS moderado IAH 19,7/h, válvula de Hakim } \\
20 \text { años antes por pseudotumor cerebral, asma con } \\
\text { hiperreactividad bronquial moderada por prueba de } \\
\text { metacolina, atelectasias pulmonares crónicas hasta } 2011 \\
\text { y en } 2013 \text { ya resueltas. }\end{array}$ \\
\hline 3 & AMDA & 73 & $\mathrm{~F}$ & RGE & Sí & No & No & $\begin{array}{l}\text { EPOC por humo de leña, RGE, uso de inhaladores } \\
\text { permanentes y tos crónica, oxígeno en casa, obesidad. }\end{array}$ \\
\hline 4 & AMFC & 59 & $\mathrm{~F}$ & Gastritis & Sí & No & No & Obesidad moderada, apnea del sueño, HTA, ¿HTP? \\
\hline 5 & DVM & 69 & M & & Sí & No & No & \\
\hline 6 & EVDG & 85 & $\mathrm{~F}$ & & Sí & Sí & No & $\begin{array}{l}\text { EPOC tipo BC por humo de leña por } 50 \text { años, oxígeno } \\
\text { dependiente, cianosis. }\end{array}$ \\
\hline 7 & IFZ & 72 & M & RGE & Sí & No & No & $\begin{array}{l}\text { EPOC, } 26 \text { pq/año, oxígeno dependiente, SAOS, } \\
\text { obesidad grado } 2 \text {. }\end{array}$ \\
\hline 8 & JTP & 82 & M & RGE & Sí & No & No & $\begin{array}{l}\text { Exposición a solventes orgánicos por } 30 \text { años, TACAR } \\
\text { de tórax: aumento de cavidades derechas, enfisema } \\
\text { centrolobulillar superior bilateral, fibrosis pulmonar panal } \\
\text { de abejas, nódulo } 20 \text { mm lóbulo superior derecho, } \\
\text { > no neoplásico, ECO-CG con aumento de cavidades } \\
\text { derechas, Doppler portal normal. }\end{array}$ \\
\hline 9 & JAGB & 84 & M & $\begin{array}{l}\text { Gastritis } \\
\text { crónica }\end{array}$ & Sí & No & No & 12 pq/año, humo de leña-agricultor, cianosis. \\
\hline 10 & JIP & 73 & M & EPOC & Sí & No & No & $\begin{array}{l}\text { EPOC-BC hace } 20 \text { años, } 40 \text { pq/año, oxígeno } 12 \text { h, } \\
\text { inhaladores, cianosis. }\end{array}$ \\
\hline
\end{tabular}


Tabla 1. Descripción de casos de pacientes con várices esofágicas proximales (continuación)

\begin{tabular}{|c|c|c|c|c|c|c|c|c|}
\hline \multirow[t]{2}{*}{ Caso } & \multirow[t]{2}{*}{ Paciente* $^{*}$} & \multirow[t]{2}{*}{ Edad } & \multirow[t]{2}{*}{ Sexo** } & \multirow{2}{*}{$\begin{array}{l}\text { Diagnóstico } \\
\text { clínico }^{+}\end{array}$} & \multicolumn{3}{|c|}{ Várices } & \multirow[t]{2}{*}{ Enfermedades de base $^{++}$} \\
\hline & & & & & Proximales & Distales & Fúndicas & \\
\hline 11 & JLEDC & 83 & M & EPOC & Sí & No & Sí & $\begin{array}{l}\text { EPID, } 25 \text { pq/año, HTP severa, fibrosis, bronquiectasias, } \\
\text { panal de abejas, engrosamiento pleural, DLCO } \\
\text { severamente disminuida, CFV restricción moderada, } \\
\text { oxígeno dependiente por hipoxemia severa. }\end{array}$ \\
\hline 12 & JMRO & 53 & M & RGE & Sí & No & No & Apnea del sueño, obesidad, HTA. \\
\hline 13 & LFQR & 69 & M & & Sí & No & No & $\begin{array}{l}\text { EPOC severa con HTP severa por ECO-CG, SAOS } \\
\text { severo IAH 39/h saturación } 75 \% \text {, obesidad, poliglobulia, } \\
\text { bicitopenia con Doppler normal portal, gammagrafía } \\
\text { sugestiva de disfunción hepática. }\end{array}$ \\
\hline 14 & LGCC & 65 & $\mathrm{~F}$ & $\begin{array}{l}\text { Gastritis } \\
\text { crónica }\end{array}$ & Sí & No & No & $\begin{array}{l}\text { HTP primaria, anticoagulación con warfarina, oxígeno } \\
\text { dependiente. }\end{array}$ \\
\hline 15 & LSGR & 81 & $\mathrm{~F}$ & $\begin{array}{l}\text { Angiodisplasia } \\
\text { esofágica, } \\
\text { presbiesófago }\end{array}$ & Sí & No & Sí & $\begin{array}{l}\text { Obesidad, HTP, EPOC por humo de leña (15 años), } \\
\text { oxígeno casa } 24 \text { h, DM-2. }\end{array}$ \\
\hline 16 & MBGG & 78 & $\mathrm{~F}$ & $\begin{array}{l}\text { Dolor } \\
\text { abdominal }\end{array}$ & Sí & No & No & $\begin{array}{l}\text { Silicosis, exposición a polvo } 30 \text { años, dependiente de } \\
\text { oxígeno, cianosis. }\end{array}$ \\
\hline 17 & $\mathrm{MH}$ & 65 & M & & Sí & No & Sí & EPOC, 30 pq/año, oxígeno dependiente. \\
\hline 18 & $\mathrm{NHA}$ & 54 & $\mathrm{~F}$ & RGE & Sí & No & No & \\
\hline 19 & PM & 73 & M & RGE & Sí & Sí & No & $\begin{array}{l}\text { EPID con fibrosis por silicosis, paquipleuritis sin } \\
\text { asbestosis, várices distales sin estigmas de sangrado. }\end{array}$ \\
\hline 20 & SPVR & 35 & $\mathrm{~F}$ & RGE & Sí & Sí & No & Secuelas de linfoma mediastino 14 años, biopsia mediastino. \\
\hline 21 & JOMR & 75 & M & $\begin{array}{c}\text { EPOC } \\
\text { Herpes zóster }\end{array}$ & Sí & No & No & $\begin{array}{l}\text { Disfonía, laringitis crónica y presbilaringe en } \\
\text { laringoscopia. Desprendimiento de retina, fumador } 90 \text { pq/ } \\
\text { año, oxígeno } 24 \mathrm{~h} \text {. }\end{array}$ \\
\hline
\end{tabular}

${ }^{*}$ Iniciales de nombres y apellidos de los pacientes. ${ }^{* *}$ Sexo: F: femenino; M: masculino.

${ }^{+}$Diagnóstico clínico. RGE: reflujo gastroesofágico; HH: hernia hiatal; EPOC: enfermedad pulmonar obstructiva crónica.

${ }^{++}$Enfermedad de base: CPAP: presión positiva controlada de la vía aérea (Continuous Positive Airway Pressure); SAOS: síndrome de apnea obstructiva del sueño; IAH: índice apnea/hipopnea; HTP: hipertensión pulmonar; BC: bronquitis crónica; TAC-AR: tomografía axial computarizada de alta resolución; ECO-CG: ecocardiograma; pq/año: número de paquetes por año; EPID: enfermedad pulmonar intersticial difusa; DM: diabetes mellitus; DLCO: difusión pulmonar al monóxido de carbono (Diffusing Capacity of the Lungs for Carbon Monoxide); CFV: curva flujo volumen

\section{Caso 2}

Paciente AJOC: mujer de 48 años remitida con diagnóstico de reflujo gastroesofágico (RGE), con antecedente de cirugía bariátrica (manga gástrica) 2 años antes por obesidad moderada, así como asma severa y apnea del sueño con síndrome de apnea hipopnea asociada a obesidad (SAHO) moderado, el cual fue corregido parcialmente con presión positiva controlada de la vía aérea (Continuous Positive Airway Pressure, CPAP).
La paciente presenta un antecedente relevante de válvula ventrículo-atrial de Hakim desde los 20 años, por causa de un pseudotumor cerebral. Entre tanto, en 2010 se encontró una várice esofágica superior, mediante una esofagogastroduodenoscopia (EGD) (Figura 1). A partir de este examen le fue descartada una patología venosa obstructiva, luego del resultado anterior, con persistencia de la várice esofágica superior y sin estigmas de sangrado o de riesgo en 2016. 
Tabla 2. Resumen de los hallazgos en pacientes con várices esofágicas proximales (Downhill varices)

\begin{tabular}{|c|c|c|c|c|c|c|c|c|}
\hline \multirow{2}{*}{$\begin{array}{l}\text { Número } \\
\text { de casos }\end{array}$} & \multirow[t]{2}{*}{ Edad* $^{*}$} & \multirow{2}{*}{$\underset{++}{\mathrm{SexO}}$} & \multirow{2}{*}{$\begin{array}{l}\text { Diagnóstico } \\
\text { clínico }^{++}\end{array}$} & \multicolumn{3}{|c|}{ Várices $^{++}$} & \multirow{2}{*}{$\begin{array}{l}\text { Otros diagnósticos } \\
\text { endoscópicos }{ }^{++}\end{array}$} & \multirow[t]{2}{*}{ Enfermedad de base ${ }^{++,} \wedge$} \\
\hline & & & & Proximales & Distales & Fúndicas & & \\
\hline \multirow[t]{8}{*}{21} & \multirow{8}{*}{$\begin{array}{l}67,95 \\
\pm 13,7 \\
(35-85)\end{array}$} & $M=11$ & RGE = 8 & 21 & 3 & 3 & Gastritis erosiva crónica $=4$ & $\mathrm{EPOC}=8$ \\
\hline & & \multirow[t]{7}{*}{$F=10$} & Herpes zóster $=1$ & & & & Hernia hiatal $=5$ & $\mathrm{SAHO}=6$ \\
\hline & & & Dolor abdominal = 1 & & & & Gastritis crónica antral = 12 & $\mathrm{HTP}=6$ \\
\hline & & & Masa abdominal $=1$ & & & & Gastritis crónica atrófica = 8 & Oxígeno dependencia $=10$ \\
\hline & & & Angiodisplasia & & & & Reflujo gastroesofágico = 3 & Obesidad $=8$ \\
\hline & & & esofágica = 1 & & & & Metaplasia intestinal $=5$ & Neumoconiosis $=2$ \\
\hline & & & Gastritis $=3$ & & & & Angiodisplasia esofágica $=1$ & $E P I D=2$ \\
\hline & & & Hernia hiatal = 1 & & & & Vólvulo gástrico = 1 & \\
\hline
\end{tabular}

*Edad en años cumplidos, promedio y desviación estándar (rango). ${ }^{+}$Sexo: F: femenino, M: masculino.

${ }^{++}$Diagnóstico clínico. Motivo de solicitud de procedimiento. RGE: reflujo gastroesofágico.

^ Enfermedad de base. EPOC: enfermedad pulmonar obstructiva crónica; SAHO: síndrome apnea-hipoventilación obesidad; HTP: hipertensión pulmonar; EPID: enfermedad pulmonar intersticial difusa.

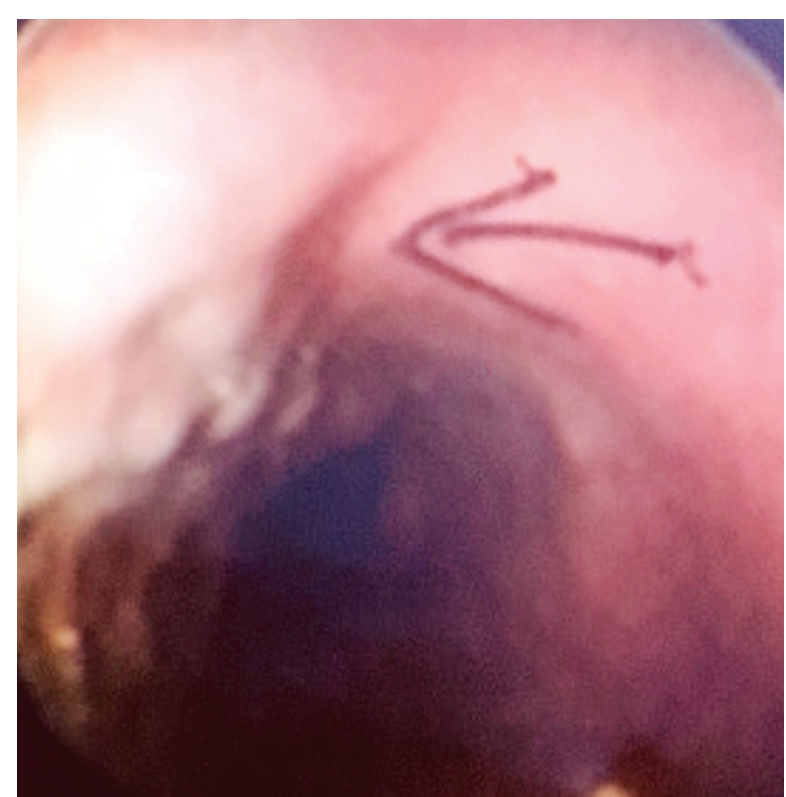

Figura 1. Caso 2: várice proximal.

\section{Caso 3}

Paciente AMDA: mujer de 73 años con diagnóstico clínico de RGE, con antecedente de EPOC por exposición al humo de leña, uso permanente de inhaladores y oxígeno suplementario en casa. En el momento del examen físico se presenta con obesidad, cianosis de piel y mucosas y abotagamiento (Figura 2). En la EGD, se encontraron várices en los 6 primeros centímetros del esófago (Figura 3) con ausencia distal de estas (Figura 4), sin estigmas de sangrado o de riesgo y gastritis crónica superficial.

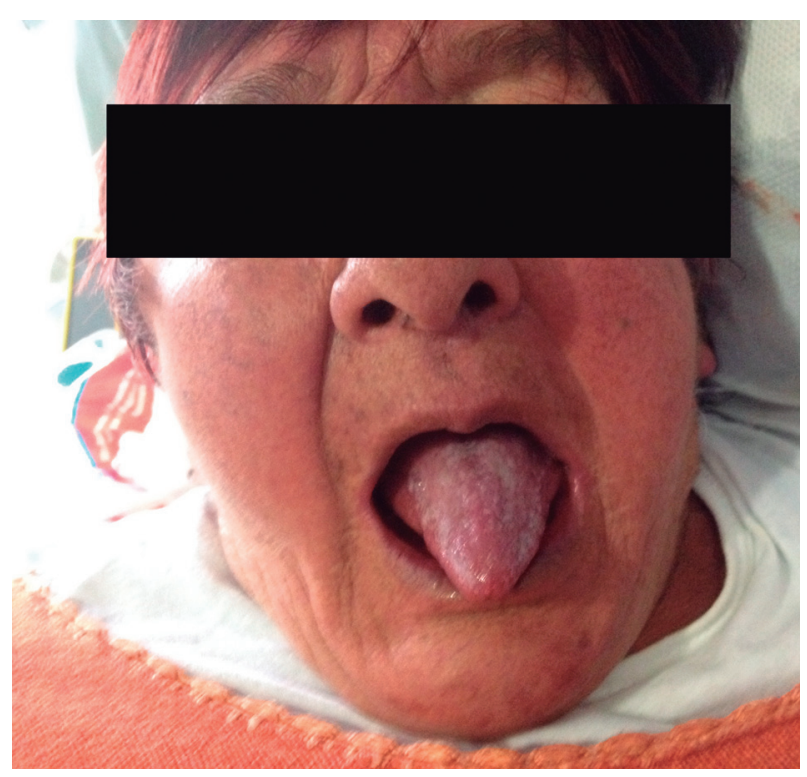

Figura 2. Caso 3: cianosis severa de piel y mucosas y abotagamiento.

\section{Caso 8}

Paciente JTP: hombre de 82 años por quien se solicitó una endoscopia, a causa de la presencia de flemas y síntomas de RGE. El paciente presenta antecedentes de exposición a solventes orgánicos por 30 años. En la tomografía axial de alta resolución (TAC-AR) de tórax se encontró un aumento del tamaño de las cavidades derechas, así como un enfisema centrolobulillar superior bilateral y fibrosis pulmonar en panal de abejas, además de un ecocardiograma (ECO-CG) con un incremento de las cavidades derechas e hipertensión pulmonar (HTP) de $49 \mathrm{~mm} \mathrm{Hg}$. Asimismo, 


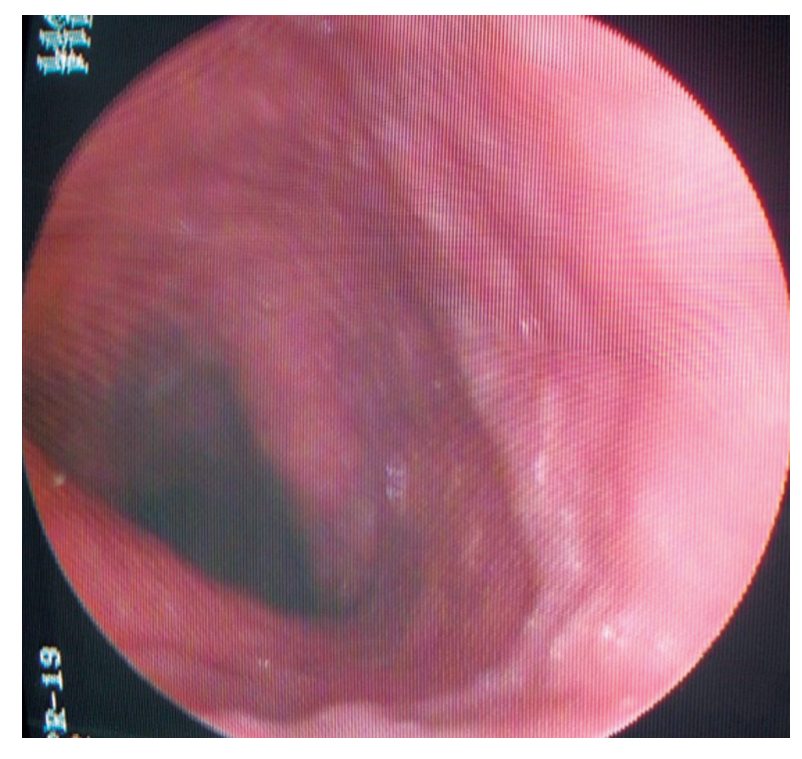

Figura 3. Caso 3: dilatación venosa esófago proximal.

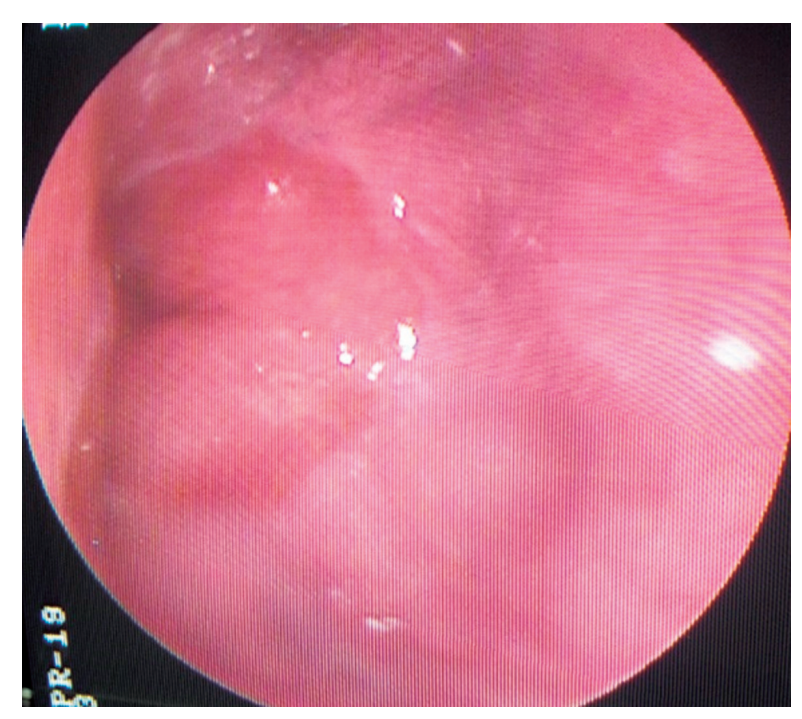

Figura 4. Caso 3: esófago distal normal, sin dilataciones venosas.

se observó un Doppler portal normal, el uso de oxígeno 18 $\mathrm{h} / \mathrm{d}$ y una polisomnografía con índice de apnea/hipopnea severo de 35,9 y ronquido en el $38 \%$ del tiempo del sueño. En la EGD se encontraron várices esofágicas superiores, sin estigmas de sangrado (Figura 5).

\section{Caso 9}

Paciente JAG: varón de 84 años, con antecedente de fumador de 12 paquetes/año, expuesto a humo de leña en

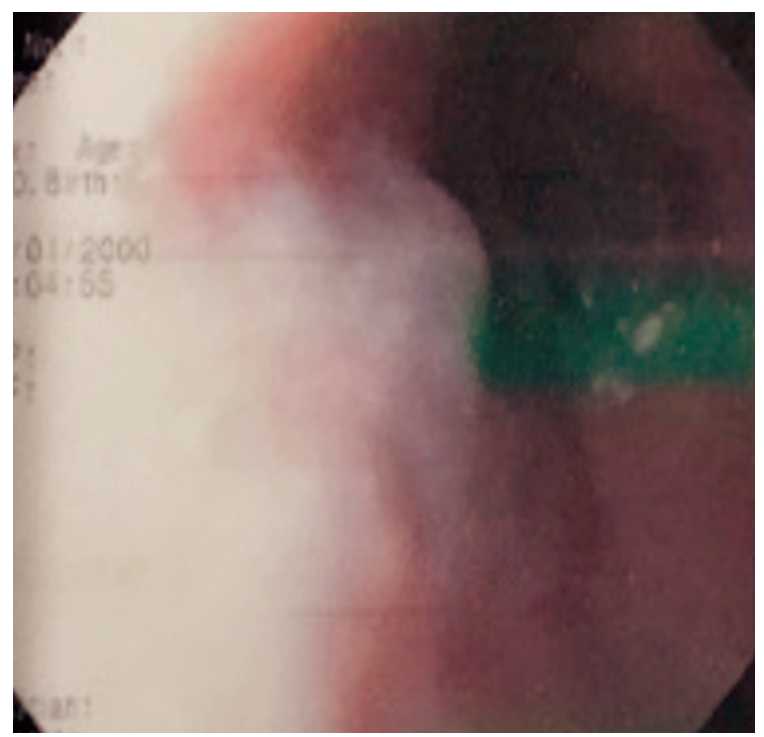

Figura 5. Caso 8: várices proximales.

quemas frecuentes en su oficio de agricultor. El sujeto es disneico y tiene una presencia importante de cianosis de la piel y mucosas y abotagamiento (Figura 6). Además, presenta un diagnóstico de gastritis crónica, razón por la cual se solicita un EGD, con hallazgo de várices en $5 \mathrm{~cm}$ proximales (Figuras 7 y 8 ) y ausencia de ellas en los tercios medio y distal, así como esofagitis péptica distal grado B (Figura 9).

\section{Caso 21}

Paciente JOMR: varón de 75 años, con antecedente de EPOC, fumador de 90 paquetes/año, dependiente de oxígeno $24 \mathrm{~h} / \mathrm{d}$, con disfonía secundaria a laringitis crónica y presbilaringe. En vista de que presenta herpes zóster y sospecha de neoplasia oculta gastrointestinal, se solicita una endoscopia, en la cual se encuentran dilataciones venosas proximales (Figuras 10 y 11 ).

\section{HALLAZGOS GENERALES}

Se presentaron 21 casos de pacientes en quienes el diagnóstico de várices esofágicas proximales fue incidental. Dichos pacientes fueron igualmente repartidos por género y edad promedio de 67,9 $\pm 13,7$ años. La endoscopia digestiva fue solicitada, principalmente, por un diagnóstico de RGE $(8 / 21)$, seguido por la gastritis crónica $(3 / 21)$, dolor epigástrico y masa abdominal durante el estudio, entre otras condiciones (Tablas 1 y 2 ).

Además de las várices en el tercio proximal, solo 3 pacientes presentaron lesiones simultáneas en el ter- 


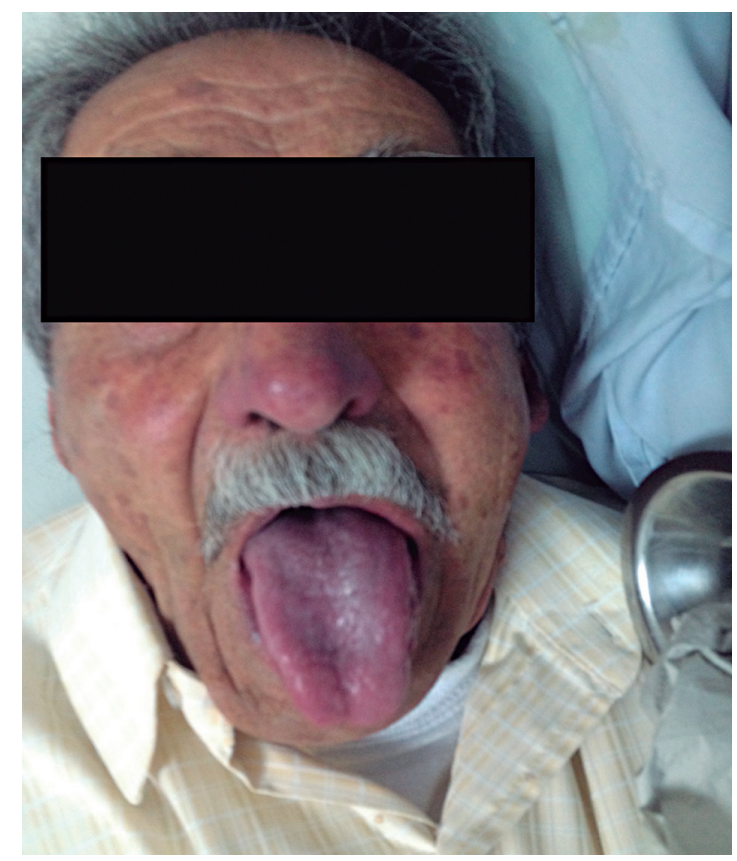

Figura 6. Caso 9: cianosis severa en la piel y mucosas, abotagamiento.

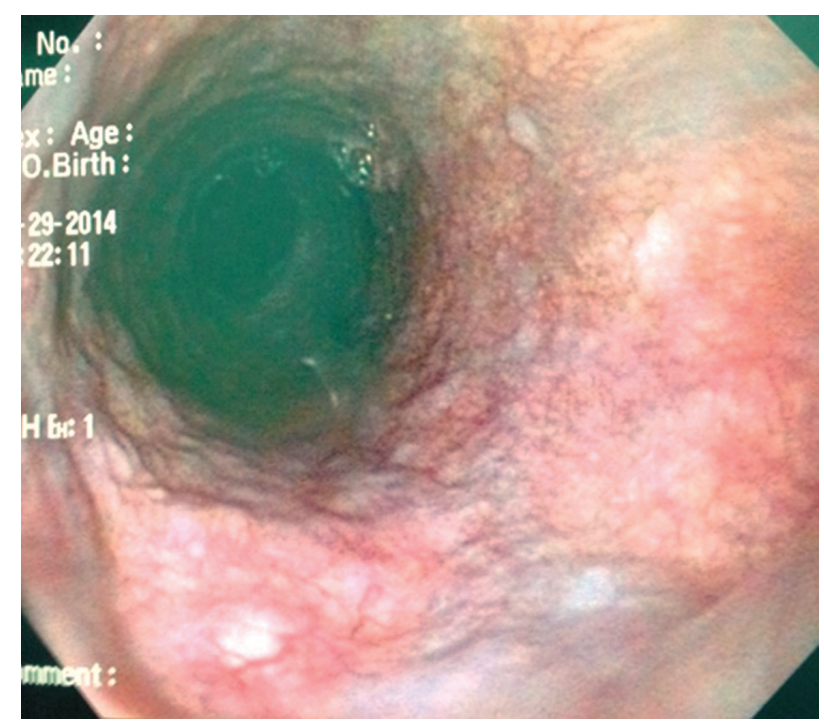

Figura 8. Caso 9: várice proximal que no se extiende al esófago medio.

cio distal, mientras que otros 3 , en el fondo gástrico en ausencia de hipertensión portal. En el 60 \%, el diagnóstico endoscópico adicional fue la gastritis crónica antral, seguida en frecuencia por la gastritis atrófica $(40 \%)$ y la hernia hiatal (25\%) (Tabla 2).

En cuanto a la enfermedad de base, se resaltó la EPOC en el $40 \%$ de los casos. Entre tanto, la HTP fue demostrada en

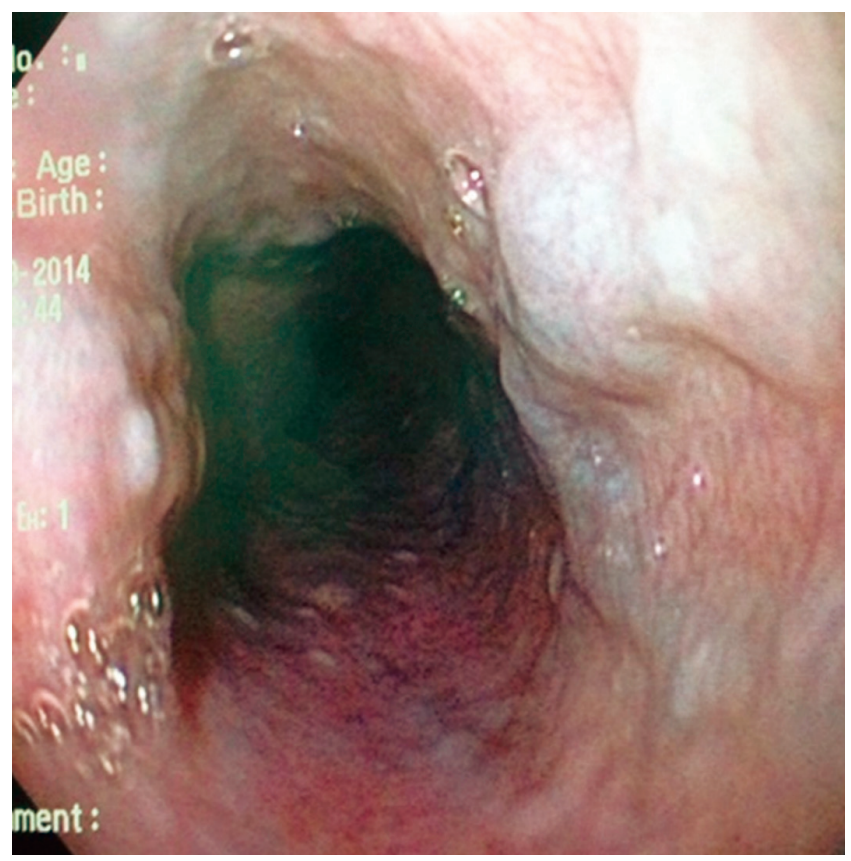

Figura 7. Caso 9: várice esofágica en el esófago proximal.

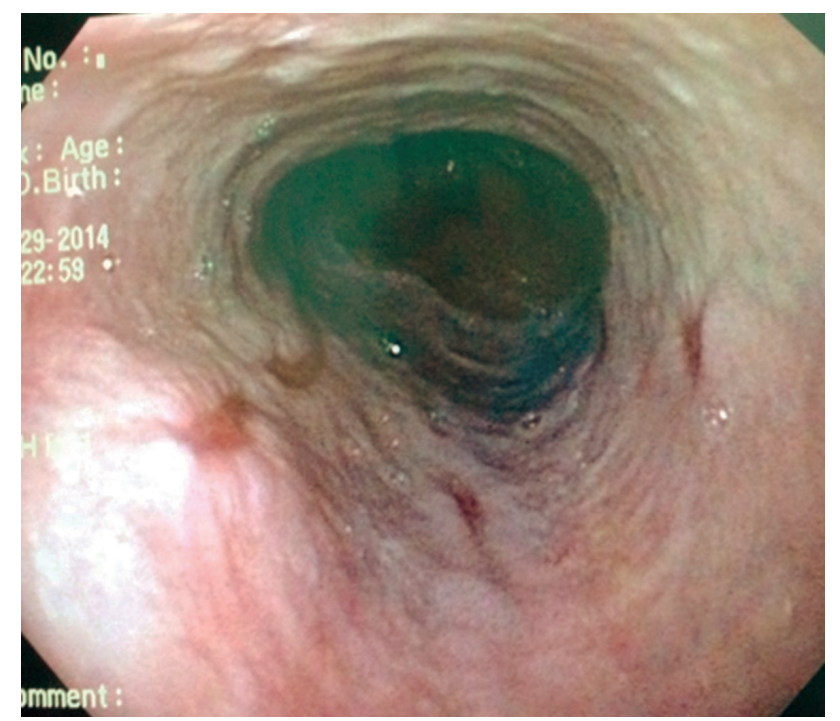

Figura 9. Caso 9: esófago distal sin várices y con esofagitis péptica erosiva grado $\mathrm{B}$.

6 pacientes $(29 \%)$ y otros $10(48 \%)$ presentaron una probabilidad de cursarla, dadas sus características clínicas y los diagnósticos asociados (Tabla 1). Se observaron 10 pacientes oxígeno dependientes ( $48 \%$ ), con obesidad moderada a severa (40\%), apnea del sueño (29\%) y enfermedad pulmonar intersticial difusa (EPID) confirmada (19\%). 


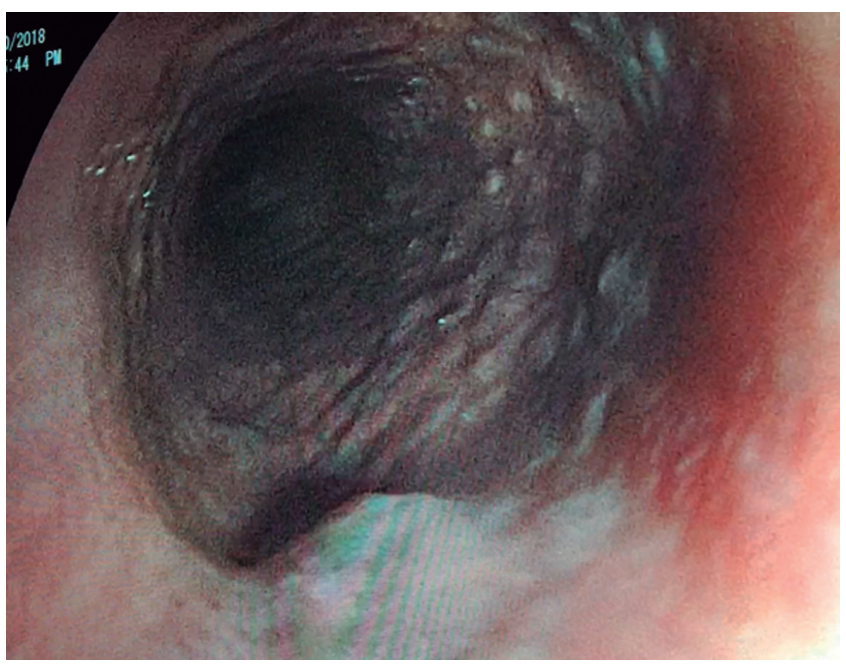

Figura 10. Caso 21: várice proximal.

\section{DISCUSIÓN}

\section{Epidemiología}

Las várices esofágicas superiores son un hallazgo poco frecuente y su denominación de Downhill varices fue acuñada por Felson en 1964 (1). Este investigador completó 30 pacientes descritos hasta ese entonces en la literatura, a partir de 3 casos. Denominó así la condición por la ocurrencia de una inversión del flujo venoso en las venas esofágicas superiores hacia abajo, en contraposición al flujo ascendente en las várices distales secundarias a la hipertensión portal: la causa principal de las várices esofágicas y el hallazgo frecuente en los servicios de endoscopia.

Una serie de 2368 casos de hemorragia de las vías digestivas altas reportó una incidencia de $0,1 \%$ de várices esofágicas superiores ( 1 de cada 908 pacientes con hemo-
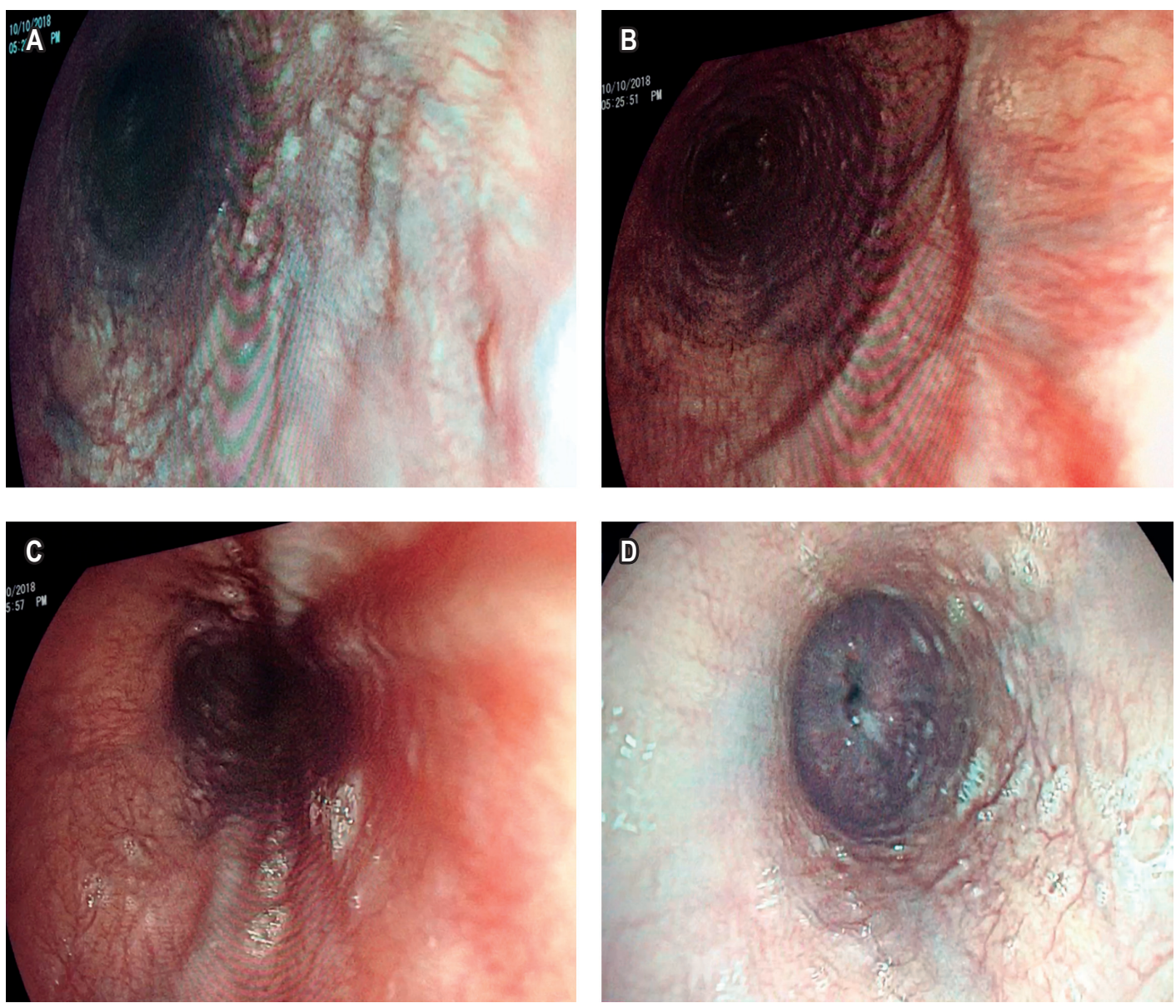

Figura 11. Caso 21. A. Várices proximales. B $\mathbf{y}$ C. Várices en el tercio medio. D. Ausencia de várices en el tercio distal. 
rragia de las vías digestivas altas) (3), mientras que las distales representaron el 10-30 \% de las causas de hemorragia digestiva alta (4).

Ayvaz y colaboradores describieron 129 casos entre 25 680 endoscopias $(0,5 \%)$, todos como hallazgo incidental y ninguno de ellos por sangrado (2); en tanto que entre los casos descritos por Felson solo en cuatro se presentó un sangrado (1). Asimismo, ninguno de los 11 sujetos con várices esofágicas proximales (halladas por TAC), de una serie de 36 pacientes, cursaron un síndrome de vena cava superior (SVCS) (5).

Como puede colegirse de los datos anteriores, la frecuencia del trastorno es muy baja y los hallazgos de endoscopia aquí reportados corresponden a sitios de diagnóstico de segundo y tercer nivel, en un período establecido entre el 1 de enero de 2011 y el 31 diciembre de 2018, para un total de 73896 endoscopias, lo cual constituye una frecuencia de $2,8 \times 10000$.

Es probable que la frecuencia de este trastorno sea superior y que, dada la ausencia de datos clínicos que sugieran dicho diagnóstico, algunos signos pasen desapercibidos ante el endoscopista. Esto, en virtud de que dichas señales suelen ser de tamaño pequeño o mediano, presentarse en los primeros centímetros del esófago y en ausencia de sangrado digestivo.

\section{Presentación clínica}

En las primeras descripciones, esta patología fue relacionada con una compresión extrínseca de la VCS, generalmente asociada a tumores mediastinales, cuyo origen frecuente es pulmonar o por la presencia de linfomas $(2,6)$.

Sin embargo, en publicaciones ulteriores se describió esta condición en patologías diversas, como el linfoma benigno gigante (7), el timoma mediastinal $(5,6,8)$ y el bocio intratorácico (9-12). Conforme se hizo frecuente el uso de dispositivos intravasculares permanentes para la realización de terapias de reemplazo renal, se describieron las trombosis secundarias de la VCS y sus ramas, con más de un centenar de casos reportados $(5,13-26)$.

Los catéteres centrales con otras indicaciones también forman parte del grupo de riesgo de desarrollo de trombosis intravasculares. Se incluyen los catéteres para nutrición parenteral total (NPT), prolongada (en casos de intestino corto) (27), quimioterapia en enfermedades malignas como leucemia aguda (28), enfermedad metastásica y otras condiciones $(2,29)$. No obstante, el común denominador es el SVCS secundario a trombosis de la VCS y sus afluentes, lo cual es ocasionado por la presencia de un cuerpo extraño intravascular y extensible a cualquier tipo de cuerpo extraño como los marcapasos transvenosos $(2,30)$.
La oclusión vascular completa, en ausencia de masa o un dispositivo que la favorezca, conduce a los mismos resultados. Tales son los casos de la enfermedad de Castleman (31), la mediastinitis fibrosante (32), la enfermedad de Behcet $(33,34)$, la venulitis sistémica (35), la hipercoagulabilidad por mutación del factor $\mathrm{V}$ de Leiden (36), el trasplante autólogo de la médula ósea con síndrome antifosfolípido (37) e incluso un caso de enfermedad congénita cardíaca (38).

Por supuesto, la trombosis de la VCS y la obstrucción de sus ramas producen el síndrome clínico característico en un número importante de los casos y conducen al diagnóstico. Sin embargo, en otros casos el diagnóstico aparece a partir de sus complicaciones, como el sangrado de las várices esofágicas. Este diagnóstico es incidental.

Otros mecanismos descritos para la aparición de várices esofágicas superiores (diferentes a la obstrucción de la VCS o sus ramas) incluyen un aumento en la presión venosa central por HTP moderada a severa; una doble lesión mitral reumática e insuficiencia tricuspídea severa (4); estenosis aórtica severa con hipertensión sistólica del ventrículo derecho (VD) e HTP severa (39); HTP severa e insuficiencia tricuspídea moderada $(2,3)$, y la presencia de EPOC severa (2).

Así pues, nuestra serie de casos aporta otro número importante a la literatura mundial. De forma singular, en el desarrollo de este trabajo solo un paciente presentó un factor obstructivo al retorno venoso, causado por una fibrosis del mediastino y como secuela del linfoma mediastinal en su adolescencia (caso 20) (Tabla 1).

Con respecto al uso de dispositivos vasculares, solo la paciente del caso 2 tenía instalada una válvula de Hakim desde los 20 años. Esta paciente no presentó una obstrucción vascular, aunque sí una patología causante de HTP, obesidad moderada y SAHO moderado, con una mejoría de estas condiciones, como consecuencia de la cirugía bariátrica. No obstante, se observó una persistencia de la várice proximal después de 6 años del diagnóstico inicial y luego de 2 años del procedimiento quirúrgico.

En nuestra serie, la mayoría de los pacientes pertenecían al grupo etario del adulto mayor (15/21: 71\%) y tanto los hombres como las mujeres de este grupo fueron afectados por igual. El $57 \%$ presentaron enfermedades pulmonares, pero la EPOC se constituyó en la más importante, de la cual solo se han descrito cuatro casos en el literatura precedente (2).

Entre tanto, la neumoconiosis (principalmente la silicosis), la neumonitis por solventes orgánicos y la EPID, como causas de várices proximales, no han sido descritas previamente y corresponden al $19 \%$ de la serie. Todas estas enfermedades comprometen el parénquima pulmonar y originan trastornos permanentes en la circulación pulmonar, así como HTP progresiva, cor pulmonale crónico y la 
consecuente elevación permanente de la presión venosa central y de sus ramas.

Otra causa de las várices proximales no descrita es la obesidad y su componente frecuente de SAHO, condiciones que son desencadenantes de HTP no obstructiva y estuvieron presentes en el $38 \%$ y el $29 \%$ de nuestros casos, respectivamente. Así pues, solo el $29 \%$ de los casos contaban con un diagnóstico clínico de HTP: la mitad de ellos eran dependientes de oxígeno y otros dos sugerían la enfermedad, dado su aspecto clínico (cianosis, poliglobulia y abotagamiento) (Figuras 2 y 6). Además, probablemente, también requerían de oxígeno suplementario.

En todos los casos, las várices fueron un hallazgo incidental y en ninguno de ellos se presentó sangrado (tampoco lo hubo previamente). Estos resultados fueron similares a los descritos en los 129 casos de Ayvaz y colaboradores (2), así como en los 11 pacientes reportados por Siegel (5).

Una de las limitaciones de nuestra serie es que no se realizaron estudios imagenológicos que nos permitiesen descartar sistemáticamente la presencia concomitante de masas mediastinales u obstrucción vascular (excepto en el caso 2). Pese a ello, clínicamente los pacientes no presentaron un SVCS u otros elementos que pudiesen generar sospecha de la presencia de tumores o de una lesión vascular y solo uno estuvo anticoagulado profilácticamente por presentar HTP primaria (caso 14). Con relación al método, este es un estudio descriptivo de casos, por lo que solo permite postular asociaciones causales.

\section{Fisiopatología}

Las venas esofágicas drenan la VCS, principalmente a través de las venas ácigos, hemiácigos y hemiácigos accesoria. En caso de obstrucción de la VCS distal a la entrada de la ácigos, el drenaje venoso de las extremidades superiores se realiza desde las venas colaterales tiroidea inferior y mediastinales hacia la hemiácigos, y desde la ácigos hacia la vena porta con flujo cefalocaudal (Downhill). Se atraviesa la vena gástrica izquierda, en cuyo caso se producen várices en todo el esófago.

Cuando la obstrucción de la VCS está por encima de la entrada de la ácigos -es decir de la VCS proximal o de los troncos braquiocefálicos o sus ramas-, el flujo colateral de estas se produce a través de las venas mamaria interna, vertebral y colaterales profundas de la ácigos, así como de las venas esofágicas superiores, igualmente con flujo cefalocaudal. De esta forma, el flujo encuentra la vía de la ácigos hacia la VCS distal a la obstrucción, lo cual genera una dilatación solamente de las venas esofágicas proximales y medias (16).

La baja frecuencia de hemorragia originada en estas várices se debe a que, solo ocasionalmente, tienen un tamaño grande y a que están localizadas en la submucosa (1-3, 5). Por esta razón se hallan menos expuestas al trauma y a la erosión péptica que sí ocurre en las várices esofágicas inferiores, en dirección caudalcefálica (uphill), dada su ubicación subepitelial (4). A estas várices esofágicas inferiores, se suma la trombocitopenia y los trastornos de coagulación, frecuentemente encontrados en pacientes con hipertensión portal.

\section{Tratamiento}

El tratamiento de esta patología debe centrarse siempre en su causa, lo cual se refiere a la escisión quirúrgica de la neoplasia mediastinal (11), el bocio intratorácico (9-11) o la corrección quirúrgica de la cardiopatía, cuando sea posible (38). Esto condiciona, generalmente, la desaparición de las várices. La terapia inmunosupresora es el tratamiento en caso de enfermedad de Behcet $(33,34)$ y de venulitis sistémica (35), mientras que la anticoagulación es el tratamiento en escenarios de trastornos de la coagulación (8, $36,37)$. Asimismo, la quimioterapia o la radioterapia son indicadas en casos de lesiones tumorales $(2,7,28,29)$.

La situación más frecuente es el hallazgo incidental de las várices sin antecedentes de sangrado, tales son los casos de la mayor serie publicada de Ayvaz y colaboradores (con 129 pacientes) (2) y la de Siegel (11). En contraste, resulta muy poco frecuente que sean diagnosticadas por sangrado variceal, como ocurre en la serie de Felson (solo 4 de 30 pacientes) (1). Esto determina una conducta conservadora con respecto a las várices esofágicas inferiores, pero naturalmente obliga al estudio de la causa desencadenante y su respectivo tratamiento.

En caso de presentarse con hemorragia de las vías digestivas, el tratamiento de elección es la colocación de bandas siempre y cuando esto sea posible técnicamente (4, 10, 14, 22-24, 27), así como también en la esclerosis, cuando la localización a nivel del cricofaríngeo imposibilita la ligadura con la banda (18), no está disponible la ligadura (21) o combinados en los casos requeridos (41).

Dentro de este contexto, se prefiere el uso de la ligadura por bandas siempre que sea posible, ya que existen descripciones de complicaciones serias como el infarto del cordón espinal por trombosis de las venas colaterales vertebrales (40) y la embolia pulmonar fatal por material empleado en la esclerosis de várices esofágicas (42).

\section{CONCLUSIONES}

Las várices esofágicas superiores son una causa rara de hemorragia de las vías digestivas altas y afectan con mayor frecuencia a los adultos mayores, sin predilección de género. En esta serie de casos, su aparición estuvo relacio- 
nada con etiologías que previamente no estaban descritas en la literatura, tales como obesidad, SAHO y EPID (por neumoconiosis o neumonitis originada por la exposición a solventes orgánicos).

Otras causas frecuentes fueron la EPOC y la HTP. En todos los casos, las várices se constituyeron en un hallazgo incidental, lo cual hace única esta serie si se compara con las existentes en la literatura indexada. En ellas, la causa más recurrente es la obstrucción de la VCS por un tumor mediastinal o por una trombosis de la VCS asociada con dispositivos intravasculares y estados de hipercoagulabilidad.

\section{Fuente de financiación}

Se declara que no existieron apoyos financieros para la ejecución y el análisis de este estudio.

\section{Observaciones}

Este estudio no ha sido presentado previamente en ninguna reunión.

\section{REFERENCIAS}

1. Felson B, Lessure AP. "Downhill" Varices of the Esophagus. Dis Chest. 1964;46:740-6. https://doi.org/10.1378/ chest.46.6.740

2. Ayvaz MA, Rakici H, Allescher HD. Are Downhill Varices an Overlooked Entity of Upper Gastrointestinal Bleedings? Gastroenterol Res Pract. 2018;2018:7638496. https://doi. org $/ 10.1155 / 2018 / 7638496$

3. Areia M, Romãozinho JM, Ferreira $\mathrm{M}$, Amaro P, Freitas D. "Downhill" varices. A rare cause of esophageal hemorrhage. Rev Esp Enferm Dig. 2006;98(5):359-61. https://doi. org/10.4321/S1130-01082006000500006

4. Harwani YP, Kumar A, Chaudhary A, Kumar M, Choudeswari PR, Kankanala VV, et al. Combined uphill and downhillvarices as a consequence of rheumatic heart disease: a unique presentation. J Clin Exp Hepatol. 2014;4(1):6365. https://doi.org/10.1016/j.jceh.2013.10.003

5. Siegel Y, Schallert E, Kuker R. Downhill esophageal varices: a prevalent complication of superior vena cava obstruction from benign and malignant causes. J Comput Assist Tomogr. 2015;39(2):149-52. https://doi.org/10.1097/ RCT. 0000000000000183

6. Subramaniam R, Madanagopalan N, Krishnan KT, Padmanabhan C. A case of anaplastic bronchogenic carcinoma with "downhill varices" of the esophagus. Dis Chest. 1967;51(5):545-9. https://doi.org/10.1378/chest.51.5.545

7. Shirakusa T, Iwasaki A, Okazaki M. Downhill esophageal varices caused by benign giant lymphoma. Case report and review of downhill varices cases in Japan. Scand J Thorac Cardiovasc Surg. 1988;22(2):135-8. https://doi. org/10.3109/14017438809105944
8. Inoue Y, Sakai S, Aoki T. Downhill oesophageal varices resulting from superior vena cava graft occlusion after resection of a thymoma. Interact Cardiovasc Thorac Surg. 2013;17(3):598600. https://doi.org/10.1093/icvts/ivt212

9. Bédard EL, Deslauriers J. Bleeding "downhill" varices: a rare complication of intrathoracic goiter. Ann Thorac Surg. 2006;81(1):358-60. https://doi.org/10.1016/j.athoracsur.2004.08.020

10. Ibis M, Ucar E, Ertugrul I, Boyvat F, Basar O, Ataseven H, et al. Inferior thyroid artery embolization for downhill varices caused by a goiter. Gastrointest Endosc. 2007;65(3):543-5. https://doi.org/10.1016/j.gie.2006.10.005

11. van der Veldt AA, Hadithi M, Paul MA, van den Berg FG, Mulder CJ, Craanen ME. An unusual cause of hematemesis: Goiter. World J Gastroenterol. 2006;12(33):5412-5415. https://doi.org/10.3748/wjg.v12.i33.5412

12. Mönkemüller K, Poppen D, Feldmann K, Ulbricht LJ. Downhill varices resulting from giant intrathoracic goiter. Endoscopy. 2010;42 Suppl 2:E40. https://doi. org $/ 10.1055 / \mathrm{s}-0029-1215290$

13. Pop A, Cutler AF. Bleeding downhill esophageal varices: a complication of upper extremity hemodialysis access. Gastrointest Endosc. 1998;47(3):299-303. https://doi. org/10.1016/S0016-5107(98)70331-1

14. Hussein FA, Mawla N, Befeler AS, Martin KJ, Lentine KL. Formation of downhill esophageal varices as a rare but serious complication of hemodialysis access: a case report and comprehensive literature review. Clin Exp Nephrol. 2008;12(5):407415. https://doi.org/10.1007/s10157-008-0055-4

15. Froilán C, Adán L, Suárez JM, Gómez S, Hernández L, Plaza $\mathrm{R}$, et al. Therapeutic approach to "downhill" varices bleeding. Gastrointest Endosc. 2008;68(5):1010-2. https://doi. org/10.1016/j.gie.2008.02.041

16. Blam ME, Kobrin S, Siegelman ES, Scotiniotis IA. "Downhill” esophageal varices as an iatrogenic complication of upper extremity hemodialysis access. Am J Gastroenterol.2002;97(1):2168. https://doi.org/10.1016/S0002-9270(01)03979-X

17. Gessel L, Alcorn J. Variants of varices: is it all "downhill" from here? Dig Dis Sci. 2015;60(2):316-9. https://doi. org/10.1007/s10620-014-3501-z

18. Gebreselassie A, Awan A, Yaqoob H, Laiyemo A. Superior Vena Cava Obstruction: A Rare Cause of Recurrent Esophageal Variceal Bleeding. Cureus. 2018;10(2):e2226. https://doi.org/10.7759/cureus.2226

19. Gopaluni S, Warwicker P. Superior vena cava obstruction presenting with epistaxis, haemoptysis and gastro-intestinal haemorrhage in two men receiving haemodialysis with central venous catheters: two case reports. J Med Case Rep. 2009;3:6180. https://doi.org/10.1186/1752-1947-3-6180

20. Muthyala U, Philipneri MD, Hussein FA, Lentine KL. Recognition of downhill esophageal varices in hemodialysis patients requires a high index of clinical suspicion. Clin Exp Nephrol. 2009;13(6):677-8. https://doi.org/10.1007/ s10157-009-0204-4

21. Ontanilla Clavijo G, Trigo Salado C, Rojas Mercedes N, Caballero Gómez JA, Rincón Gatica A, Alcívar-Vasquez JM, 
et al. Downhill varices: an uncommon cause of upper gastrointestinal bleeding. Rev Esp Enferm Dig. 2016;108(7):440442. https://doi.org/10.17235/reed.2016.3697/2015

22. Pillai U, RoopkiranjotK,Lakshminarayan N,Balabhadrapatruni K, Gebregeorgis W, Kissner P. Downhill varices secondary to HeRO graft-related SVC syndrome. Semin Dial. 2013;26(5):E47-9. https://doi.org/10.1111/sdi.12078

23. Raghavapuram $S$, George N, Girotra $M$, Siddique $S$, Tharian B. Downhill esophageal varices: unusual cause of hematemesis. VideoGIE. 2017;2(9):231-232. https://doi. org/10.1016/j.vgie.2017.02.005

24. Berkowitz JC, Bhusal S, Desai D, Cerulli MA, Inamdar S. Downhill Esophageal Varices Associated With Central Venous Catheter-Related Thrombosis Managed With Endoscopic and Surgical Therapy. ACG Case Rep J. 2016;3(4):e102. https://doi.org/10.14309/crj.2016.75

25. Loudin M, Anderson S, Schlansky B. Bleeding 'downhill' esophageal varices associated with benign superior vena cava obstruction: case report and literature review. BMC Gastroenterol. 2016;16(1):134. https://doi.org/10.1186/ s12876-016-0548-7

26. Nayudu SK, Dev A, Kanneganti K. “Downhill” Esophageal Varices due to Dialysis Catheter-Induced Superior Vena Caval Occlusion: A Rare Cause of Upper Gastrointestinal Bleeding. Case Rep Gastrointest Med. 2013;2013:830796. https://doi.org/10.1155/2013/830796

27. Lim EJ, Stella DL, Russell DM. Torrential upper gastrointestinal bleeding from 'downhill' oesophageal varices complicating long term central venous access for total parenteral nutrition. Frontline Gastroenterol. 2010;1(2):118-120. https://doi.org/10.1136/fg.2010.001354

28. Yeung AK, Guilcher GM, deBruyn JC. Conservative Management of Downhill Esophageal Varices Secondary to Central Line-related Thrombosis After Hematopoietic Stem Cell Transplant.J Pediatr Hematol Oncol. 2015;37(7):e4246. https://doi.org/10.1097/MPH.0000000000000373

29. Yasar B, Abut E. A case of mediastinal fibrosis due to radiotherapy and 'downhill' esophageal varices: a rare cause of upper gastrointestinal bleeding. Clin J Gastroenterol. 2015;8(2):736. https://doi.org/10.1007/s12328-015-0555-1

30. Basar N, Cagli K, Basar O, Sen N, Gurel OM, Akpinar I, et al. Upper-extremity deep vein thrombosis and downhill esophageal varices caused by long-term pacemaker implantation. Tex Heart Inst J. 2010;37(6):714-716.

31. SerinE, OzerB, GümürdülüY,Yildirim T, Barutçu O,Boyacioglu S. A case of Castleman's disease with "downhill" varices in the absence of superior vena cava obstruction. Endoscopy. 2002;34(2):160-2. https://doi.org/10.1055/s-2002-19840
32. Basaranoglu M, Ozdemir S, Celik AF, Senturk H, Akin P. A case of fibrosing mediastinitis with obstruction of superior vena cava and downhill esophageal varices: a rare cause of upper gastrointestinal hemorrhage. J Clin Gastroenterol. 1999;28(3):268-70. https://doi.org/10.1097/00004836199904000-00021

33. Orikasa H, Ejiri Y, Suzuki S, Ishikawa H, Miyata M, Obara K, et al. A case of Behçet's disease with occlusion of both caval veins and "downhill" esophageal varices. J Gastroenterol. 1994;29(4):506-10. https://doi.org/10.1007/BF02361251

34. EnnaiferR,B'chirHamzaouiS,LarbiT,RomdhaneH,Abdallah M, Bel Hadj N, et al. Downhill oesophageal variceal bleeding: A rare complication in Behçet's disease-related superior vena cava syndrome. Arab J Gastroenterol. 2015;16(1):36-8. https://doi.org/10.1016/j.ajg.2015.02.003

35. Maton PN, Allison DJ, Chadwick VS. “Downhill” esophageal varices and occlusion of superior and inferior vena cavas due to a systemic venulitis. J Clin Gastroenterol. 1985;7(4):3317. https://doi.org/10.1097/00004836-198508000-00013

36. Gómez-Aldana AJ, Gómez-Zuleta. Varices esofágicas en Downhill secundarias a trombosis de vena cava superior por déficit de factor V. Rev Gastroenterol Mex. 2017;82(2):179180. https://doi.org/10.1016/j.rgmx.2016.04.007

37. Vorlop E, Zaidman J, Moss SF. Clinical challenges and images in GI. Downhill esophageal varices secondary to superior vena cava occlusion. Gastroenterology. 2008;135(6):1863, 2158. https://doi.org/10.1053/j.gastro.2008.10.069

38. Malloy L, Jensen M, Bishop W, Divekar A. "Downhill” esophageal varices in congenital heart disease. J Pediatr Gastroenterol Nutr. 2013;56(2):e9-11. https://doi. org/10.1097/MPG.0b013e31824b5fff

39. Gholam S, Ghazala S, Pokhrel B, Desai AP. A Rare Case of Downhill Esophageal Varices in the Absence of Superior Vena Cava Obstruction. Am J Gastroenterol. 2017;112(3):413. https://doi.org/10.1038/ajg.2016.489

40. Heller SL, Meyer JR, Russell EJ. Spinal cord venous infarction following endoscopic sclerotherapy for esophageal varices. Neurology. 1996;47(4):1081-5. https://doi. org/10.1212/WNL.47.4.1081

41. Nguyen LP, Sriratanaviriyakul N, Sandrock C. A Rare but Reversible Cause of Hematemesis: "Downhill” Esophageal Varices. Case Rep Crit Care. 2016;2016:2370109. https:// doi.org/10.1155/2016/2370109

42. Tsokos M, Bartel A, Schoel R, Rabenhorst G, Schwerk WB. [Fatal pulmonary embolism after endoscopic embolization of downhill esophageal varix]. Dtsch Med Wochenschr. 1998;123(22):691-5. https://doi. org/10.1055/s-2007-1024039 